\section{Penetration of topically applied levofloxacin into eyes with thin-wall filtering bleb after trabeculectomy}

\begin{abstract}
Purpose To investigate the comparative penetration of $0.3 \%$ levofloxacin eye drops into the aqueous humour between cataract patients with or without (control) thin-wall filtering blebs.

Methods One drop of $\mathbf{0 . 3 \%}$ levofloxacin was administered to the eyes at 30 -min intervals for $3.5 \mathrm{~h}$ before phacoemulcification for both groups. Aqueous humour samples (0.1-0.2 ml) were aspirated during surgery. The concentration of levofloxacin in the aqueous humour was determined by high-performance liquid chromatography. The Student's $t$-test, Pearson correlation, and $\chi^{2}$ test were used to compare the data of the two groups. A $P<0.05$ was required for results to be considered statistically significant.

Results The levofloxacin concentration in the aqueous humour was significantly increased $(P<0.0001)$ in the bleb (mean + SD: $3.7 \pm 2.3 \mu \mathrm{g} /$ ml) vs control group $(0.4 \pm 0.2 \mu \mathrm{g} / \mathrm{ml})$. Intraocular pressure and the bleb area were not correlated with levofloxacin concentration. Conclusion The presence of thin-wall filtering blebs increases intraocular penetration of topically administered levofloxacin.

Eye (2008) 22, 666-670; doi:10.1038/sj.eye.6702712; published online 2 March 2007
\end{abstract}

Keywords: thin-wall filtering bleb; levofloxacin; intraocular penetration; aqueous humour; trabeculectomy

\section{Introduction}

Trabeculectomy creates a fistula between the anterior chamber and the subconjunctival
LY Qiao ${ }^{1,2}$, NL Wang2, YB Liang², SQ Zhu², $\mathrm{XH} \mathrm{Wan}^{2}$ and $\mathrm{P}-\mathrm{Y} \mathrm{Lee}^{2}$ tissues, thereby creating a new channel for aqueous humour drainage. Eventually, the aqueous humour penetrates the conjunctiva and flows into the tear film on the eye's surface. The most common problem associated with this surgery is the growth of scar tissue, which causes an obstruction in this artificial channel and blocks aqueous humour drainage. Various antifibrotic medications, such as mitomycin $\mathrm{C}$ (MMC) and 5-fluorouracil (5-FU), can be used to prevent the excessive growth of scar tissue and improve surgical success. ${ }^{1-7}$ The most evident risk factor for late bleb leaks, bleb ruptures, and infections is the intraoperative or postoperative use of antimetabolites. After administration of antimetabolites, blebs tend to be more ischemic and thinner. ${ }^{8-13}$ The aqueous humour can egress from the filtering bleb to the conjunctival sac through the wall of a thin-wall bleb, that is without a focal leak, owing to the dysfunctional conjunctival barrier. ${ }^{8,14}$ Thus, we have

hypothesized that topical administration of eye drops could penetrate the wall of the thin-wall bleb into the intrableb space, and then directly and more readily could enter the aqueous humour through the artificial subscleral channel. As a result, the intraocular penetration of eye drops may enhance the effects of therapeutic or unfavourable agents. The effect of the thin-wall filtering bleb on levofloxacin penetration into the aqueous humour has never been investigated.

Levofloxacin is a synthetic broad spectrum antibacterial agent for topical, oral, and intravenous administration. Chemically, levofloxacin, a chiral fluorinated carboxyquinolone, is the optical s-(-) isomer of ofloxacin. It is generally twice as potent as ofloxacin when tested in vitro. Levofloxacin is active against most aerobic Gram-positive and 
Gram-negative organisms and demonstrates moderate activity against anaerobes. Drug penetration into body tissues and fluids is rapid and widespread after oral administration. ${ }^{15}$

The purpose of this study was to investigate the comparative penetration of topically applied $0.3 \%$ levofloxacin into the aqueous humour of cataract patients with thin-wall filtering blebs as compared with that of control patients without filtering blebs.

\section{Materials and methods}

The study involved 15 cataractous eyes (15 patients) with primary open-angle or angle-closure glaucoma. The patients had successful filtering surgery with thin-wall filtering blebs and were not taking topical medications for $4.4 \pm 2.6$ years (mean \pm SD) after trabeculectomy combined with 5-FU postoperatively or MMC intraoperatively (bleb group). The control group consisted of 15 cataractous eyes (15 patients) with no history of ocular surgeries or other ocular diseases. Eyes were excluded in this study if they had lacrimal, conjunctival, corneal disorders, or Seidelpositive aqueous leakage of the bleb wall. ${ }^{14,16}$ Eyes with thin-wall filtering blebs were defined as those with a postoperative intraocular pressure (IOP) lower than the preoperative value by $>3 \mathrm{mmHg}$ without medication and a bleb wall that was thin and avascular. A moistened sterile strip gently applied to the bleb surface for $15 \mathrm{~s}$ did not demonstrate leakage by slit-lamp observation ${ }^{14,16}$ and the cornea was not stained with $2 \%$ fluorescein. The aqueous humour outflow route was patent as demonstrated by UBM in the bleb eyes.

All patients were recruited from the Tongren Eye Center, Beijing Tongren Hospital (Beijing, China). Informed consent was obtained from each patient involved in this study according to the principles outlined in the Helsinki Declaration, and the study was approved by the Ethics Committee of the Beijing Tongren Eye Center.

IOP was measured with a Goldmann applanation tonometer. Three IOP readings were taken at $5 \mathrm{~min}$ intervals and averaged. The largest horizontal and vertical diameter of the filtering blebs was measured with a ruler. The area of each bleb was calculated by multiplication of the two measurements.

Cataract surgery (phacoemulcification) was performed on all patients. Topical administration of one drop of 0.3\% levofloxacin (Zheng Da Fu Rui Da Pharmacy, Shan Dong Province, China) was administered at $30 \mathrm{~min}$ intervals for $3.5 \mathrm{~h}$ before surgery. At the beginning of the cataract operation, $0.1-0.2 \mathrm{ml}$ of aqueous humour was withdrawn from the anterior chamber via the peripheral cornea using a tuberculin syringe. The samples were stored at $-80^{\circ} \mathrm{C}$ before assay.

Concentration of levofloxacin in aqueous humour was analysed using high-performance liquid chromatography. The analytical column was a $4.6 \times 250 \mathrm{~mm}$ in internal diameter cartridge packed with $5 \mu \mathrm{m}$ Diamonsil C18. All experiments were performed at a flow rate of $0.7 \mathrm{ml} / \mathrm{min}$ at ambient temperature. The mobile phase consisted of methanol-acetonitrile-1\% acetic acid (10:10:80,v/v/v) and $5 \mathrm{mmol} / \mathrm{l}$ tetrabutylammonium bromide. The examined wavelengths were set to $294 \mathrm{~nm}$ for levofloxacin concentrations and determined against a calibration curve constructed from their standard concentrations. Concentrations were calculated from peak values and expressed as micrograms of drug per $\mathrm{ml}$ of aqueous humour.

The Student's $t$-test, Pearson correlation, and $\chi^{2}$ test were used to compare the data of the two groups. A $P<0.05$ (two-tailed test) was required for results to be considered statistically significant.

\section{Results}

A total of 30 eyes (30 patients) with cataract consisting of 15 eyes (15 patients) with glaucoma and previously successful filtering surgery and thin-wall filtering blebs and 15 eyes ( 15 patients) without glaucoma were included in this report. The mean age was $55.7 \pm 13.2$ years (mean $\pm S D$ ) in the bleb group and $65.5 \pm 5.9$ years in the control group. Patient demographic data are described in the Table 1.

The aqueous humour level (Mean + SD) of levofloxacin was $3.7 \pm 2.3 \mu \mathrm{g} / \mathrm{ml}$, ranging from 1.3 to $9.2 \mu \mathrm{g} / \mathrm{ml}$, in the bleb group and $0.4 \pm 0.2 \mu \mathrm{g} / \mathrm{ml}$, ranging from 0.1 to $0.7 \mu \mathrm{g} / \mathrm{ml}$, in the control group. Accordingly, the mean concentration of levofloxacin in aqueous humour of the bleb group was nine times that of the control group $(P<0.0001)$ (Figure 1).

The mean IOP before cataract surgery was $9.8 \pm 2.8 \mathrm{mmHg}$, ranging from 4.0 to $13.0 \mathrm{mmHg}$, in the bleb group and $14.9 \pm 2.1 \mathrm{mmHg}$, ranging from 11.0 to $19.0 \mathrm{mmHg}$, in the control group. Analysis of these values reveals that the mean IOP in bleb group was significantly $(P<0.0001)$ lower than that of the control

Table 1 Demographic data of patients

\begin{tabular}{lccc}
\hline Variable & Bleb group & Control group & P-value \\
\hline Age (years) & $55.7 \pm 13.2^{\mathrm{a}}$ & $65.5 \pm 5.9$ & 0.017 \\
Gender & & & \\
Male & 10 & 9 & 1.000 \\
Female & 5 & 6 & \\
\hline
\end{tabular}

aSignificant difference as compared with control group ( $\chi^{2}$ test). 


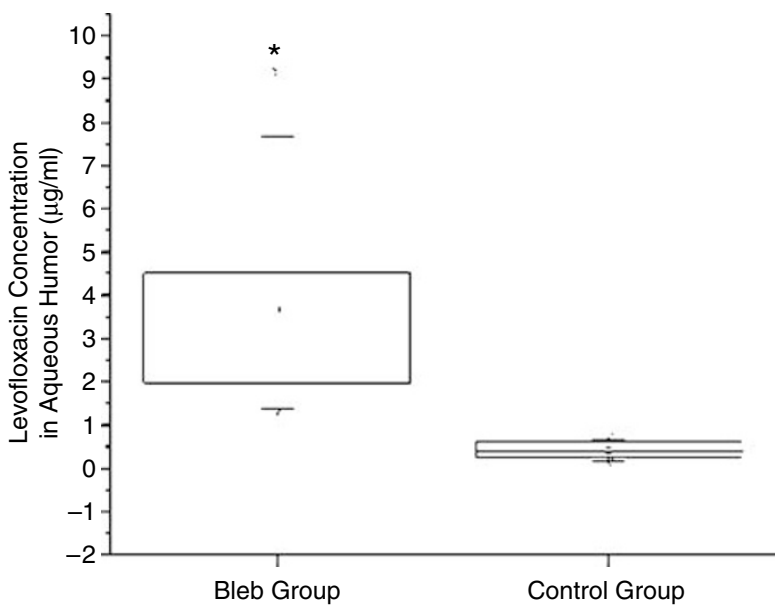

Figure 1 The concentration of levofloxacin in aqueous humour Box-and-whisker plots show the distribution of the levofloxacin concentration in aqueous humour in the bleb group and the control group. The mean score is indicated by the horizontal lines through the box. Asterisk indicate statistically significant increase in levofloxacin concentration compared with the control group (Student's $t$-test, $P<0.0001$ ).

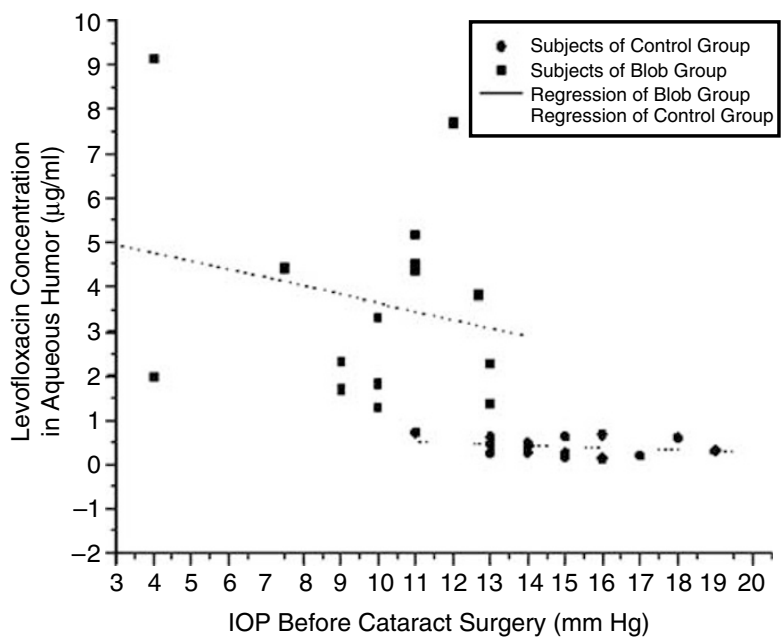

Figure 2 Correlation between levofloxacin concentration in aqueous humour and IOP before cataract surgery in the bleb group and the control group. Levofloxacin concentrations in aqueous humour were not significantly correlated with the IOP before cataract surgery in the bleb group $(r=-0.277, P=0.208)$ or the control group $(r=-0.278, P=0.316)$.

group. However, no statistically significant correlation was observed between the IOP and the concentration of levofloxacin in aqueous humour for the bleb group $(r=-0.277, P=0.208)$ or the control group $(r=-0.278$, $P=0.316$ ) (Figure 2).

The mean area of the filtering blebs was $33.7 \pm 14.8 \mathrm{~mm}^{2}$, ranging from 20.0 to $63.0 \mathrm{~mm}^{2}$, and was not significantly related to changes in the concentration

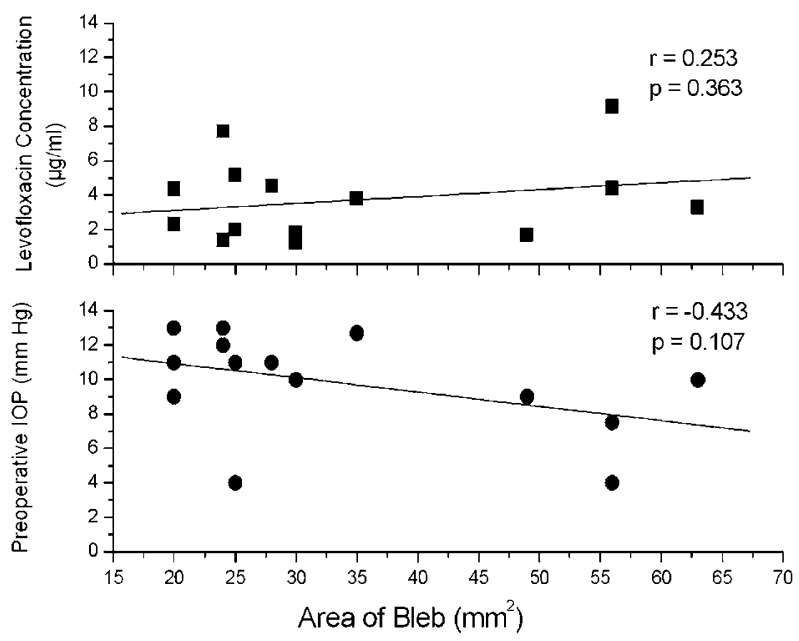

Figure 3 Correlation between the area of filtering bleb and levofloxacin concentration in aqueous humour or IOP before cataract surgery in the bleb group.

of levofloxacin in aqueous humour $(r=0.253, P=0.363)$. Nor was there a significant correlation between the area of the filtering blebs and IOP before cataract surgery $(r=-0.433, P=0.107)$ for the bleb group (Figure 3).

\section{Discussion}

After glaucoma filtering surgery, especially in the case of thin-wall and avascular blebs, some patients may need topically applied drugs to treat blebitis, endophthalmitis, or other ocular diseases. Owing to the dysfunctional conjunctival barrier of a thin-wall bleb, eye drops may penetrate the wall of the thin avascular bleb into the intrableb space. As a result, these drugs have the potential to enter the aqueous humour directly through the artificial subscleral channel in greater concentrations.

As an example of this eventuality, we show in this study that the concentration of levofloxacin in aqueous humour was significantly $(P<0.0001)$ increased in the bleb group. These findings are comparable to the study of Matsuo et al, ${ }^{17}$ who reported that fluorescein concentration in the anterior chamber was significantly higher in the bleb group than in the topical-medication and untreated control group.

In our study, the mean aqueous humour level of levofloxacin was $3.7 \pm 2.3 \mu \mathrm{g} / \mathrm{ml}$ in the bleb group and $0.4 \pm 0.2 \mu \mathrm{g} / \mathrm{ml}$ in the control group after topical treatment with a total of eight drops of $0.3 \%$ levofloxacin in cataractous eyes. The data of our control group is similar to that of previous studies that have demonstrated a mean aqueous humour concentration of levofloxacin of $1.00 \pm 0.48$ or $0.73 \mu \mathrm{g} / \mathrm{ml}$ after topical treatment with either three or four drops of $0.5 \%$ 
levofloxacin in cataractous eyes without filtering blebs. ${ }^{18,19}$

Cantor $e t a^{20}$ reported topical antibiotic treatment yielded mean concentrations of ofloxacin $=0.75 \mu \mathrm{g} / \mathrm{ml}$, and ciprofloxacin $=0.21 \mu \mathrm{g} / \mathrm{ml}$, in aqueous of eyes with functioning filtering blebs, but the objective in their study was to determine which antibacterial agents had greater penetration into the aqueous humour of eyes with functioning filtering blebs. They did not compare directly the antibacterial concentration in the aqueous humour between eyes with and without functioning blebs. In their report, fluoroquinolone concentrations in the aqueous humour of eyes without filtering blebs compared with were the data from previous reports. They concluded that the presence of the filtering bleb (in the absence of corneal and conjunctival leakage) does not increase antibacterial drug penetration into the aqueous humour. In our study, we found the mean concentration of levofloxacin in aqueous humour was nine times higher in the thin-wall bleb group than in the control group $(P<0.0001)$. The indirect nature of the comparison as performed by Cantor $e t a l^{20}$ may have resulted in the selection of a functioning bleb with a horned and thicker bleb wall, accounting for the difference between theirs and our present results.

There are two routes for an eye drop getting into the eye, one is through the cornea and the other involves is a non-cornea passage. In normal eyes, most topical drugs enter the eye mainly through the corneal route. ${ }^{21}$ Levofloxacin is a moderate lipid-soluble antibacterial with good corneal penetrability. ${ }^{22}$ In the cornea route, the corneal epithelia are the main barrier. ${ }^{21}$ Some topical drugs, such as antifibrotic agents, anti-glaucoma eyedrops or anaesthesia, influence the corneal epithelia integrity. In our study, all eyes had intact corneal epithelia as indicated by a negative $2 \%$ fluorescein test. Our findings that levofloxacin in the bleb group reaches a greater than nine-fold concentration in aqueous humour as compared with controls, imply that the drug penetrates the thin and avascular bleb wall with a dysfunctional conjunctival barrier into the intrableb space, and then directly enter the aqueous humour through the artificial subscleral channel.

The age of the control group was significantly higher ( $P=0.017)$ than that of the bleb group, which agrees with the result of the Advanced Glaucoma Investigate Survey (AGIS), that cataract incidence is increased after filtration surgery and it forms earlier. ${ }^{23}$ It is probable that corneal epithelia are not decreased with ageing, ${ }^{24}$ suggesting that age may not be a critical factor with regard to the topical drug penetration into eyes. Accordingly, the differences in age between the two groups may not significantly affect our results. On the contrary, the increased age of the control group would favour increased penetration through the aged corneal epithelia, so the concentration differences in levofloxacin difference between two groups of the present report may actually be underestimated.

In our study, the IOP of the bleb group was significantly $(P<0.0001)$ lower than that of the control group. As levofloxacin is a lipid-soluble antibacterial, it can theoretically penetrate biologic tissues easier. In this way, levofloxacin penetration of the bleb wall is determined by the difference in concentration between conjunctiva and intrableb space, and is not the result of the IOP. The IOP in both groups was not correlated with the concentration of levofloxacin in the aqueous humour. This result indicated that the levofloxacin concentration in aqueous humour was not influenced by the IOP in the two groups. We also found that the filtering bleb area was not correlated with the concentration of levofloxacin in the aqueous humour or IOP before cataract surgery in the bleb group. It is possible that the levofloxacin concentration in the aqueous humour achieved a steady state concentration. Under such conditions, the bleb area would not be closely related to levofloxacin concentration. An additional consideration is the potential for a diminished precision with the use of a ruler to measure the largest horizontal and vertical diameter for calculating the area of blebs.

It is known that continued use of steroids can induce cataracts, and the toxic effect is related to the period of steroid topical administration and steroid concentration in the aqueous humour. ${ }^{25}$ Based on our results, the thinwall bleb may represent an alternative route for topical drugs to penetrate into the eye. Thus, topical administration of drugs to the eyes with thin-wall blebs should be performed with caution. It may be necessary to decrease the concentration of some eye drops (eg steroids, levoflaxacin) to eyes with thin-wall blebs, to avoid any toxic effects or over-medication of the ocular tissues.

The present results clearly show that the achievable levofloxacin concentrations can vary markedly in the bleb vs non-bleb group. In particular, our findings suggest that the thin-wall bleb may be a pathway that increases the penetration of topically administrated levofloxacin into the eyes.

\section{Acknowledgements}

We thank Ms Zhongli Huang from Instrumental Analysis \& Research Center of Sun Yatsen University, Guangzhou, China, for her analysis of the concentration of levofloxacin by HPLC. We also thank Professor Steven M Podos, MD, from Department of Ophthalmology, Mount Sinai School of Medicine, New York, USA, for his guidance and correcting this paper. 


\section{References}

1 The Fluorouracil Filtering Surgery Study Group. Fluorouracil filtering surgery study one-year follow-up. Am J Ophthalmol 1989; 108: 625-635.

2 The Fluorouracil Filtering Surgery Study Group. Three-year follow-up of the fluorouracil filtering surgery study. Am J Ophthalmol 1993; 115: 82-92.

3 The Fluorouracil Filtering Surgery Study Group. Five-year follow-up of the fluorouracil filtering surgery study. Am J Ophthalmol 1996; 121: 349-366.

4 Kitazawa Y, Kawase K, Matsushita H, Minobe M. Trabeculectomy with mitomycin. A comparative study with fluorouracil. Arch Ophthalmol 1991; 109: 1693-1698.

5 Liebmann JM, Ritch R, Marmor M, Nunez J, Wolner B. Initial 5-fluorouracil trabeculectomy in uncomplicated glaucoma. Ophthalmology 1991; 98: 1036-1041.

6 Araie M, Shoji N, Shirato S, Nakano Y. Postoperative subconjunctival 5-fluorouracil injections and success probability of trabeculectomy in Japanese: results of 5-year follow-up. Jpn J Ophthalmol 1992; 36: 158-168.

7 Cheung JC, Wright MM, Murali S, Pederson JE. Intermediate-term outcome of variable dose mitomycin C filtering surgery. Ophthalmology 1997; 104: 143-149.

8 Sihota R, Dada T, Gupta SD, Sharma S, Arora R, Agarwal HC. Conjunctival dysfunction and mitomycin C-induced hypotony. J Glaucoma 2000; 9: 392-397.

9 Nuyts RM, Felten PC, Pels E, Langerhorst CT, Geijssen HC, Grossniklaus HE et al. Histopathologic effects of mitomycin $\mathrm{C}$ after trabeculectomy in human glaucomatous eyes with persistent hypotony. Am J Opthalmol 1994; 118: 225-237.

10 Mietz H, Brunner R, Addicks K, Krieglstein GK. Histopathology of an avascular filtering bleb after trabeculectomy with mitomycin-C. J Glaucoma 1993; 2 266-270.

11 Greenfield DS, Parrish RK. Bleb rupture following filtering surgery with mitomycin-C: clinicopathologic correlations. Opthalmic Surg Lasers 1996; 27: 876-877.

12 Belyea DA, Dan JA, Stamper RL, Lieberman MF, Spencer WH. Late onset of sequential multifocal bleb leaks after glaucoma filtration surgery with 5-fluorouracil and mitomycin C. Am J Opthalmol 1997; 124: 40-45.
13 Shields MB, Scroggs MW, Sloop CM, Simmons RB. Clinical and histopathologic observations concerning hypotony after trabeculectomy with adjunctive mitomycin C. Am J Ophthalmol 1993; 116: 673-683.

14 Matsuo H, Tomidokoro A, Suzuki Y, Shirato S, Araie M. Late-onset transconjunctival oozing and point leak of aqueous humor from filtering bleb after trabeculectomy. Am J Opthalmol 2002; 133: 456-462.

15 Davis R, Bryson HM. Levofloxacin. A review of its antibacterial activity, pharmacokinetics and therapeutic efficacy. Drugs 1994; 47: 677-700.

16 Siegfried CJ, Grewal RK, Karalekas D, Rosenberg LF, Krupin T. Marked intraocular pressure rise complicating intrableb autologous blood injection. Arch Ophthalmol 1996; 114: 492-493.

17 Matsuo H, Tomidokoro A, Araie M. Penetration of topically applied fluorescein in to eyes with avascular filtering bleb after trabeculectomy. Am J Ophthalmol 2005; 140: 742-744.

18 Yamada M, Mochizuki H, Yamada K, Kawai M, Mashima Y. Aqueous humor levels of topically applied levofloxacin in human eyes. Curr Rye Res 2002; 24: 403-406.

19 Colin J, Simonpoli S, Geldsetzer K, Ropo A. Corneal penetration of levofloxacin into the human aqueous humour: a comparison with ciprofloxacin. Acta Ophthalmol Scand 2003; 81: 611-613.

20 Cantor LB, Donnenfeld E, Katz J, Gee WL, Finley CD, Lakhani VK. Penetration of ofloxacin and ciprofloxacin into the aqueous humors of eyes with functioning filtering blebs. Arch Ophthalmol 2001; 119: 1254-1257.

21 Chen Z. Ocular Clinic Phamacology. Chemical Industry Press: Beijing, 2002, p 9.

22 Chen Z. Ocular Clinic Phamacology. Chemical Industry Press: Beijing, 2002, p 110.

23 The AIGS Investigators. The advanced glaucoma intervention study: risk of cataract formation after trabeculectomy. Arch Opthalmol 2001; 119: 1771-1779.

24 Mustonen RK, McDonald MB, Srivannaboon S, Tan AL, Doubrava MW, Kim CK. Normal human corneal cell populations evaluated by in vivo scanning slit confocal microscopy. Cornea 1998; 17: 485-492.

25 Li F. Ophthalmology. People's Medical Publishing House: Beijing, 1997, p 1573. 